\title{
Modelling and model verification of an autonomous threshold sensor for humidity measurements
}

\author{
Nikolai Gulnizkij and Gerald Gerlach \\ Solid-State Electronics Laboratory, TU Dresden, Dresden, 01062, Germany \\ Correspondence: Nikolai Gulnizkij (nikolai.gulnizkij@tu-dresden.de)
}

Received: 12 July 2019 - Revised: 23 October 2019 - Accepted: 29 October 2019 - Published: 6 January 2020

\begin{abstract}
Autonomous sensors that receive their energy from an energy harvester or directly from the environment have the potential to save energy for applications in numerous sectors. For humidity sensing, the swelling behaviour of a water vapour-sensitive hydrogel is used. To trigger an electrical contact the mechanical energy is taken from the hydrogel swelling by the bimorph effect. As long as a defined threshold is not reached, the electrical microcontact remains open. By passing the threshold value, a switching will be triggered causing the closure of the contact. This sensor principle does not need any electrical power supply because the switching power is provided directly by the surrounding humidity as quantity to be measured. For the description of the deflection versus the hydrogel pattern of such a sensor, a model was developed by means of the beam theory. Goal of the model was to derive design guidelines for the dimensioning of the sensors bending plate and the patterned hydrogel layer. Experiments then should show the applicability of the model approaches. The deflection of the bending plate depends on the ratio between the lengths of the uncoated and the coated part of the bending plate where the maximum occurs at a ratio of ca. 0.5 . The swelling behaviour of the hydrogel shows a high sensitivity with regard to slight changes in relative humidity. This can be used for humidity threshold sensors that open and close microcontacts with respect to very small changes in relative humidity. To avoid voltage peaks and arcs during the switching process that could arise and destroy the microcontact, a bistable characteristic with hysteresis is needed. Experiments demonstrate the feasibility of this concept and are in good agreement with the modelling results.
\end{abstract}

\section{Motivation}

The recent progress in industrial production, building automation, automobile technology and many other fields leads to an increasing demand on sensors and sensor systems that combine data acquisition and signal processing. Such sensor systems have achieved above-average growth of more than $6 \%$ per year (AMA, 2018) in the sensor and measurement technology industry in recent decades. Temperature and humidity are the most important measured quantities. However, in process control $70 \%$ of all sensors are used as threshold switches and in building monitoring more than $90 \%$. In Bellmann et al. (2015), a novel approach for a non-powered humidity threshold switch based on the BIZEPS (binary zeropower sensor) principle was proposed (see also Frank et al., 2012; Rangelow et al., 2009; Rittersma, 2002). With this principle, the energy is taken directly from the measured variable. In this work, a humidity threshold sensor switch is considered, where the moisture-dependent swelling of a hydrogel, which is part of a bimorph bending plate, is utilized. The switching hysteresis required to achieve an abrupt closing and opening of the contacts can be realized by applying a pre-deflection as well as an axial compression force. To prove this concept, in this work here a simple macro device is considered where both the pre-stress and the pre-deflection are applied by a screw acting axially on an elastically suspended bending beam coated with a humidity-sensitive hydrogel layer.

\section{Setup and working principle}

Figure 1 shows the general setup and working principle of MEMS-based moisture threshold switches (Huang et al., 2015; Dai et al., 2013). The silicon-based bending plate with 


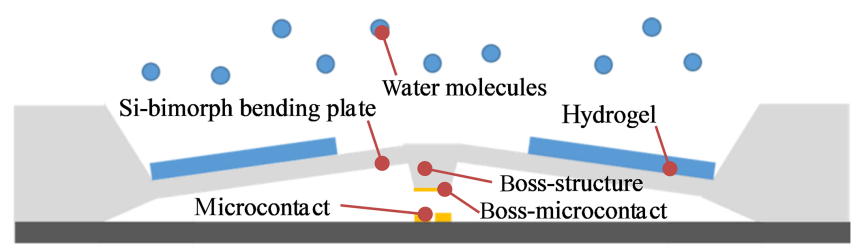

(a)

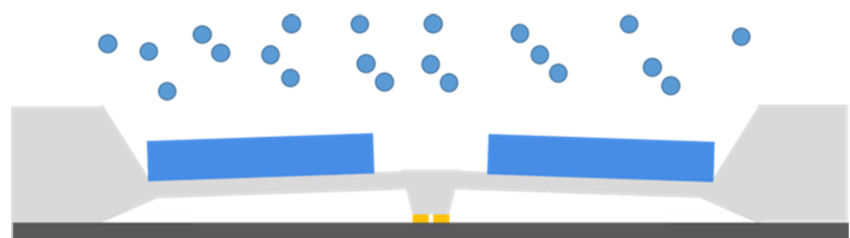

(b)

Figure 1. Humidity threshold sensor switch, consisting of a bimorph bending plate with boss structure, a patterned hydrogel layer and microcontacts, (a) open, (b) closed.

a thickness of ca. $2 \times 10^{-5} \mathrm{~m}$ is located in the middle of a silicon die of ca. $5 \times 10^{-4} \mathrm{~m}$ thickness. In the middle, the thin plate comprises a thicker part - the so-called boss structure - that carries one part of the contact pair to be closed or opened. It ensures that the microcontact remains level and flat even if the bending plate is deflected. The humiditydependent swelling of the hydrogel-coated silicon plate deflects the rigid centre of the bending plate. The water vapour uptake and, hence, the swelling state depend on the humidity of the surrounding air. When swelling, the hydrogel performs mechanical work and, thus, deflects the bending plate mechanically. That means that the energy is supplied by the environment and provides the opportunity to operate the sensor autonomously without any electrical energy supply. Because the swelling and de-swelling are reversible processes, the device can be used both for switching on and off the contacts, respectively.

\section{Model without axial forces}

To calculate the deflection as a function of the hydrogel pattern, a simple mechanical model is used based on the beam theory for monomorph and bimorph beams (Marschner et al., 2014). Figure 2 shows the setup of the sensor and the corresponding mechanical model. The setup consists of the hydrogel-coated part of the bending beam with the torsional compliance $n_{\mathrm{B}}$ and the uncoated part of the bending beam with the torsional compliance $n_{\mathrm{P}}$ :

$n_{\mathrm{P}}=\frac{12 l_{P}}{E_{1} h_{1}^{3} b}$,

$n_{\mathrm{B}}=\frac{1}{b} \frac{12\left(1+\frac{E_{2} h_{2}}{E_{1} h_{1}}\right) l_{B}}{E_{1} h_{1}^{3}\left(1+\frac{E_{2}^{2} h_{2}}{E_{1}^{2} h_{1}}+\frac{E_{2}}{E_{1}}\left(4 \frac{h_{2}}{h_{1}}+6 \frac{h_{2}^{2}}{h_{1}^{2}}+4 \frac{h_{2}^{3}}{h_{1}^{3}}\right)\right)}$.
Hydrogels are much softer than silicon, steel or other spring materials, so that $E_{2} \ll E_{1}$ applies. Considering that the hydrogel coating and the bending beam have the same thickness $h_{1}=h_{2}$, Eq. (2) yields a good approximation:

$n_{\mathrm{B}}=\frac{12 l_{B}}{E_{1} h_{1}^{3} b}$.

The hydrogel-coated part of the setup acts as a torsional moment source $M_{\mathrm{RH}}$ when the relative humidity causes a swelling strain $\varepsilon_{\mathrm{RH}}$ :

$M_{\mathrm{RH}}=\frac{b}{2} \frac{E_{1} E_{2} h_{1} h_{2}\left(h_{1}+h_{2}\right)}{E_{1} h_{1}+E_{2} h_{2}} \cdot \varepsilon_{\mathrm{RH}} \approx E_{2} h_{1}^{2} b \cdot \varepsilon_{\mathrm{RH}}$.

The swelling strain $\varepsilon_{\mathrm{RH}}$ results from the expansion coefficient $\alpha_{\mathrm{RH}}$ of the hydrogel due to relative humidity $\varphi_{\mathrm{RH}}$ :

$\varepsilon_{\mathrm{RH}}=\alpha_{\mathrm{RH}} \varphi_{\mathrm{RH}}$.

$M_{\mathrm{RH}}$ has to deflect both $n_{\mathrm{B}}$ and $n_{\mathrm{P}}$, so that the overall torsional compliance $n$ amounts to

$n=\frac{n_{\mathrm{B}} \cdot n_{\mathrm{P}}}{n_{\mathrm{B}}+n_{\mathrm{P}}}$.

According to Fig. 2c the deflection $s$ results from the angle $\varphi$ and the simplification that both parts of the setup are composed of lumped torsional springs with the deflected length $\left(l_{B}+l_{P}\right)=l / 2$ :

$s=\frac{l}{2} \varphi=\frac{l}{2} n M_{\mathrm{RH}}$.

By inserting Eqs. (1), (2), (4) and (6), it yields the following:

$s=6 \frac{E_{2} l^{2}}{E_{1} h_{1}} \cdot \frac{l_{B}}{l}\left(1-\frac{l_{B}}{l}\right) \cdot \varepsilon_{\mathrm{RH}}$.

Introduction of the degree of coverage $x$

$x=\frac{l_{B}}{l}$

leads to

$s=6 \frac{E_{2} l^{2}}{E_{1} h} \cdot x(1-x) \cdot \varepsilon_{\mathrm{RH}}$.

Equation (10) shows that a maximum deflection can be achieved

- by a high ratio $e_{2} / e_{1}$ of Young's moduli of hydrogel and substrate (bending plate: silicon, steel),

- by thin bending plates,

- by large swelling strains $\varepsilon_{\text {rh }}$ and expansion coefficients $\alpha_{\mathrm{rh}}$, respectively. 


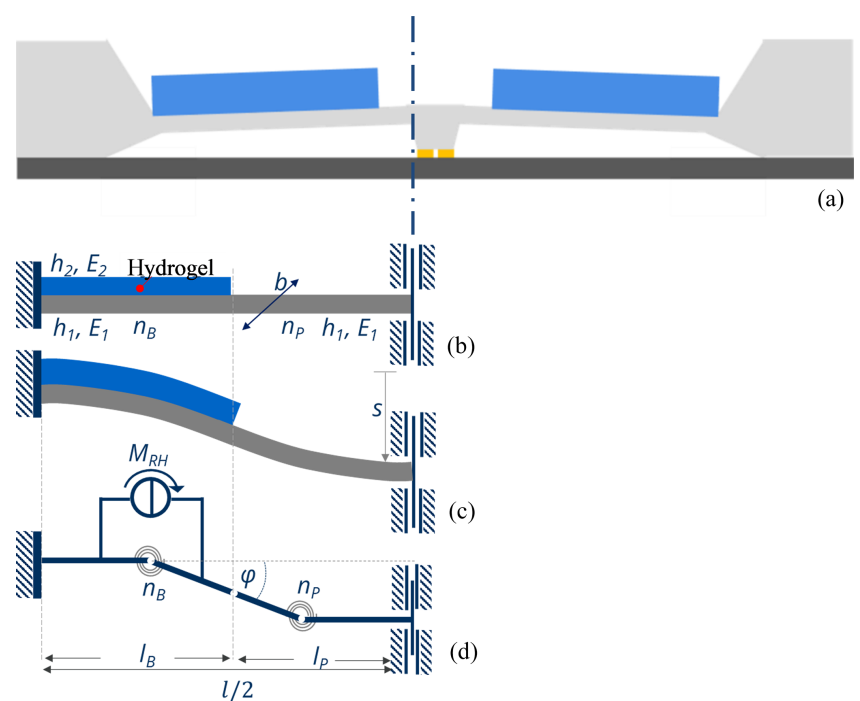

Figure 2. Model of the sensor switch of Fig. 1 without axial force, (a) setup, (b) bending beam partially coated with a hydrogel layer, (c) deflected sensor due to relative humidity changes, and (d) corresponding mechanical model. Shown is half of the sensor due to symmetry reasons (left: rigid clamping, right: vertically deflectable edge of the boss structure). $n_{B}, n_{P}$ torsional compliance of the bending beam with and without hydrogel, respectively, $l$ length, $l_{B}$ length of the hydrogel-covered part, $s$ deflection, $\varphi$ angle of deflection at the transition point between the hydrogel-coated and the uncoated part, $M_{\mathrm{RH}}$ torsional moment due to the hydrogel swelling, $b$ width, $h$ thickness. Indices: 1 substrate (silicon, steel), 2 hydrogel, $p$ substrate, $b$ hydrogel/hydrogel-coated.

A maximum deflection $s_{\max }$ is reached if $\mathrm{d} s / \mathrm{d} x=0$, i.e. if the degree $x$ of hydrogel coverage amounts to

$x_{\mathrm{opt}}=0.5$

leading to

$s_{\max }=\frac{3}{2} \frac{E_{2}}{E_{1}} l \frac{l}{h} \cdot \varepsilon_{\mathrm{RH}}$.

For practical applications, one has to take into account

- that the model of Fig. 2d considers just a bending beam instead of usually used two-dimensional plates,

- that the model of Fig. 2b simplifies the setup by considering two lumped torsional springs and

- Eqs. (3) and (4) assume $E_{2} \ll E_{1}$ and $h_{1}=h_{2}=h$.

For that reason, the optimum value $x_{\mathrm{opt}}=0.5$ of Eq. (11) might differ slightly. More general, a maximum deflection is obtained when the compliances $n_{\mathrm{B}}$ and $n_{\mathrm{P}}$ of the hydrogelcovered and non-covered parts are equal.

\section{Model with axial forces}

To avoid arcing and damage of the microcontact, the sensor has to switch quickly between the closed and opened state.
From this, the switching characteristic needs a hysteresis This can be achieved when the bending beam is axially compressed by means of an axial force or by applying a source of expansion. To consider switching hysteresis properties, the model of Fig. 2 is complemented by an axial force $F_{\mathrm{A}}$ and an axially acting translational spring $n_{\mathrm{T}}$ (Fig. 3).

Figure 3 shows the corresponding model, based on the beam theory, of an axially compressed bending beam (Timoshenko and Gere, 2012). Here, for reasons of simplicity, the condition $l_{B}=l_{P}=l / 4$ is assumed. From Fig. 3a, the deflection $s_{0}$ and the deflection angle $\varphi_{0}$ of the bending beam result from a source of expansion that leads to a length expansion of the bending beam part from length $l_{A}$ to length $l_{B}$ (Fig. 3b):

$l_{A}=\frac{l}{4}+s_{0} \frac{l}{4} \frac{1}{\cos \left(\varphi_{0}\right)}$,

$l_{B}=\frac{l}{4} \frac{1}{\cos \left(\varphi-\varphi_{0}\right)}$,

where $\varphi_{0}$ and $\varphi$ are the pre-deflection angle and the change of angle.

The resulting compression $\Delta s$ yields

$\Delta s=l_{B}-l_{A}=\frac{l}{4}\left(\frac{1}{\cos \left(\varphi-\varphi_{0}\right)}-\frac{1}{\cos \left(\varphi_{0}\right)}\right)$,

leading to an axial force

$F_{A}=\frac{\Delta s}{n}=\frac{1}{n} \frac{l}{4}\left(\frac{1}{\cos \left(\varphi-\varphi_{0}\right)}-\frac{1}{\cos \left(\varphi_{0}\right)}\right)$.

For the derivation of the bending moment equation at equilibrium state, $F_{A}$ was considered with its two vectorial components:

$F_{A x}=F_{A} \cos \left(\varphi-\varphi_{0}\right)$,

$F_{A y}=F_{A} \sin \left(\varphi-\varphi_{0}\right)$.

During swelling, the hydrogel-covered part acts as a bimorph providing a bending moment source $M_{\mathrm{RH}}$ depending on the humidity change. From Fig. $3 \mathrm{~b}$, the length change of $l_{B}$ along the $x$ direction results from the deflection angle $\varphi-\varphi_{0}$. The balance of torques yields together with Eqs. (16)-(18):

$M_{\mathrm{RH}}-F_{A y} \frac{l}{4}+F_{A x} \frac{l}{4} \tan \left(\varphi-\varphi_{0}\right)=0$.

For small deflection angles, the trigonometric functions in Eq. (19) can be expressed with the corresponding first terms of the Taylor series:

$$
\begin{aligned}
& \sin (x)=x-\frac{x^{3}}{3 !}, \\
& \cos (x)=1-\frac{x^{2}}{2 !}, \\
& \tan (x)=x+\frac{x^{3}}{3},
\end{aligned}
$$



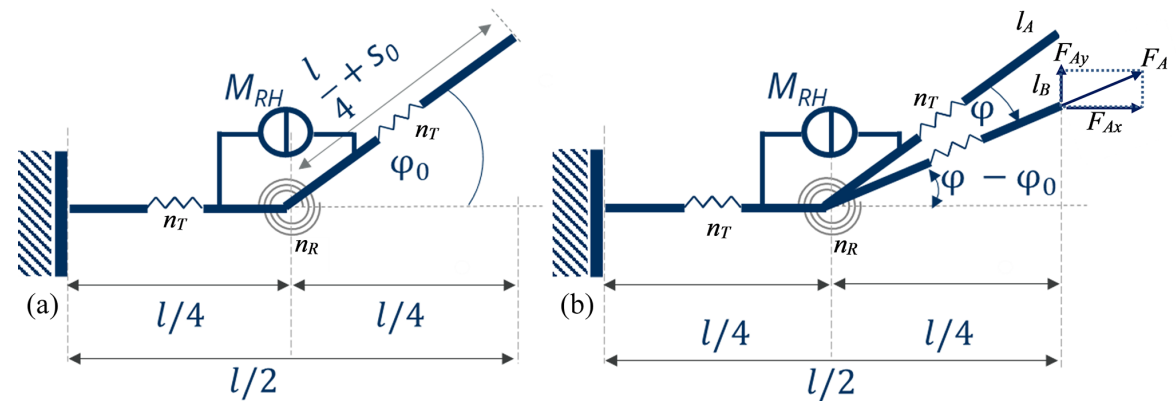

Figure 3. Mechanical model for a pre-deflected bending beam with length $l / 2$, pre-deflection angle $\varphi_{0}$, torsional spring $n_{R}$, translational spring $n_{\mathrm{T}}$ and humidity-dependent bending moment $M_{\mathrm{RH}}(\mathbf{a})$ without and (b) with axial compressive force $F_{\mathrm{A}}$.

so that Eq. (18) becomes

$M_{\mathrm{RH}}-F_{A} \frac{l}{4}\left(\varphi-\varphi_{0}\right)+F_{A} \frac{l}{4}\left(\varphi-\varphi_{0}\right)^{3}=0$.

Regarding Eq. (4), $M_{\mathrm{RH}}$ is proportional to $\varepsilon_{\mathrm{RH}}$ and, hence, to relative humidity $\varphi_{\mathrm{RH}}$, where $\varphi_{\mathrm{RH}}$ is proportional to the deflection $s$. This allows us to re-write Eq. (23):

$M_{\mathrm{RH}}-A\left(\varphi-\varphi_{0}\right)+B\left(\varphi-\varphi_{0}\right)^{3}=0$

and respectively

$\varphi_{\mathrm{RH}}-A^{*} s+B^{*} s^{3}=0$.

$A$ and $B$ as well as $A^{*}$ and $B^{*}$ are coefficients to be calculated from $M_{\mathrm{RH}}$ and $F_{A}$ via Eqs. (4), (5) and (15). Figure $4 \mathrm{a}$ shows the curve progression of $\varphi_{\mathrm{RH}}$ versus $\left(\varphi-\varphi_{0}\right)$ for varying parameters $A^{*}$ and $B^{*}$. For $A^{*}=1$ and $B^{*}=0$, the curve progression shows a linear dependency of $\varphi_{\mathrm{RH}}$ on $\left(\varphi-\varphi_{0}\right)$. For $A^{*}=1$ and $0.2<B^{*}<0.9$, a hysteresis occurs and for $A^{*}=1$ and $B^{*}=1$ a switching without a hysteresis was found. As a consequence, by varying both the bending beam geometry and the material parameters, the shape of the curves and the width of the hysteresis can be adjusted. In Fig. 4b, the S-shaped curve progression with switching hysteresis is shown for $A^{*}=1$ and $B^{*}=0.5$ depending on the relative humidity with the switching points from $s_{1}$ to $s_{2}$ and from $s_{3}$ to $s_{4}$, respectively, and the curve progression without switching hysteresis for $B^{*}=1$ and $B^{*}=0$.

\section{Fabrication of a sensor switch with switching hysteresis}

To ensure that the electrical microcontacts are reliably closed and opened, oscillations between the opened and closed states as well as local electric field strength peaks that are too high and associated arcs have to be avoided. This can be achieved by implementing a switching hysteresis between two stable states (Chen et al., 2008).

To demonstrate the concept, a beam-like sensor switch was manufactured from sheet metal, an easy-to-process material, with Young's modulus of $2.06 \times 10^{11} \mathrm{~N} \mathrm{~m}^{-2}$ (Fig. 5a)
Table 1. Material and geometry parameters of the beam-like sensor switch with switching hysteresis (DIN EN 10270-1, 2012; Bellmann, 2016).

\begin{tabular}{lrr}
\hline Parameter & $\begin{array}{r}\text { Sheet metal } \\
\left(n_{\mathrm{B}}, n_{\mathrm{P}}\right)\end{array}$ & Hydrogel \\
\hline Length $(\mathrm{m})$ & 0.025 & 0.025 \\
Width $(\mathrm{m})$ & 0.012 & 0.012 \\
Thickness (m) & $3 \times 10^{-5}$ & $3 \times 10^{-5}$ \\
Young's modulus & $2.06 \times 10^{11}$ & $3.5 \times 10^{9}$ at $5 \% \mathrm{RH}$ \\
$\left(\mathrm{N} \mathrm{m}^{-2}\right)$ & & $3 \times 10^{8}$ at $90 \% \mathrm{RH}$ \\
\hline
\end{tabular}

(DIN EN 10270-1, 2012). As shown in Fig. 5b, the bending beam was fixed in a fixation and axially clamped by means of an adjusting screw.

The humidity-sensitive hydrogel layer (thickness $3 \times$ $10^{-5} \mathrm{~m}$ ) was structured on the top of the metal sheet. To achieve sufficient adhesion the metal surface was pretreated by means of a chemical cleaning process and an adhesion-promoting agent. For that, the bending beam was cleaned in a solution made of distilled $\mathrm{H}_{2} \mathrm{O}, 25 \%$ ammonia and $25 \%$ peroxide $(2: 1: 1)$ at $358.15 \mathrm{~K}$ for $900 \mathrm{~s}$. Afterwards, the bending beam was pre-treated in a plasma etching system (VTD: DREVA 450) with oxygen plasma at a pressure of $15 \mathrm{~Pa}$ at $400 \mathrm{~W}$ for $300 \mathrm{~s}$. Then a $3 \mathrm{~mL}$ solution of the adhesion-promoter agent made of $0.9 \mathrm{~mL}$ (3Aminopropyl)triethoxysilane (APTES) and $99 \mathrm{~mL} \mathrm{H}_{2} \mathrm{O}$ was applied to the plasma-treated surface and heated in an oven at $373.15 \mathrm{~K}$ for $600 \mathrm{~s}$ (Gartmann, et al., 2010).

The moisture-sensitive hydrogel was made from a blend of $15 \mathrm{wt} \%$ poly(vinyl alcohol) (PVA, molecular mass $M_{w}=$ $89000 \ldots 98000$ ) and $7.5 \mathrm{wt} \%$ poly(acrylic acid) (PAA, $\left.M_{w}=450000\right)$ in a mass ratio of $4: 1$. Both materials were purchased from Sigma-Aldrich. The powders were solved in deionized water and mixed for several hours for PAA at $343.15 \mathrm{~K}$ and for PVA at $353.15 \mathrm{~K}$ until homogenous solutions were formed. Then both polymer solutions were mixed for several hours at $323.15 \mathrm{~K}$ to form the hydrogel blend (Jose et al., 2014). The hydrogel layer was deposited by a 

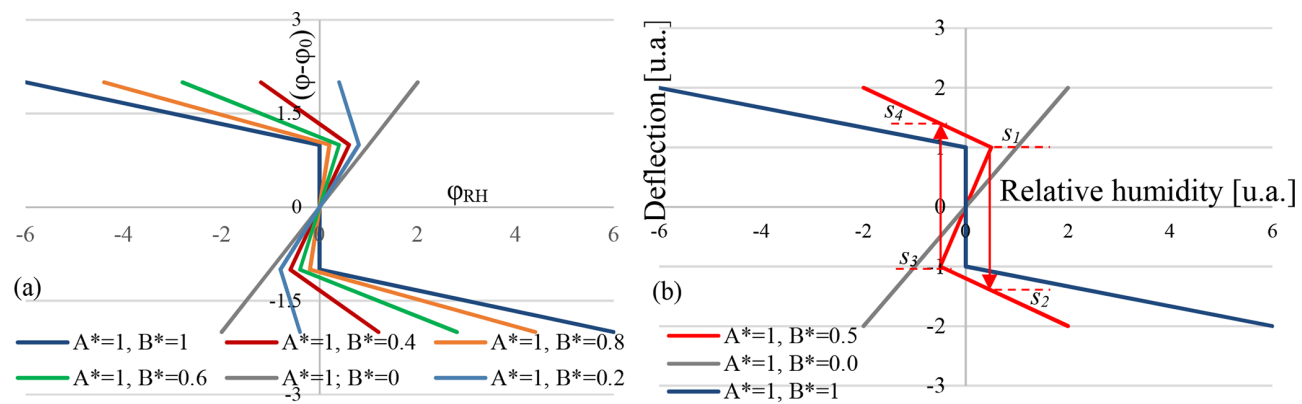

Figure 4. Calculated curves for (a) deflection $s$ and (b) angle $\varphi-\varphi_{0}$ versus relative humidity $\varphi_{\mathrm{RH}}$. The switching points $\left(s_{1} \rightarrow s_{2} ; s_{3} \rightarrow s_{4}\right)$ are marked.
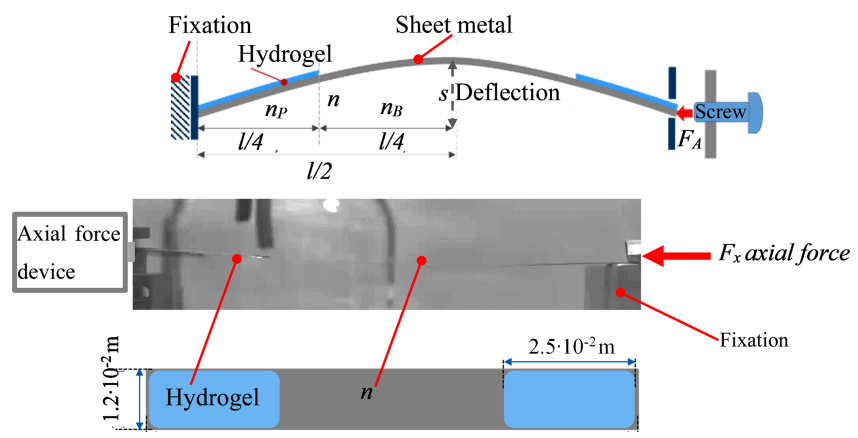

Figure 5. Beam-like sensor switch with switching hysteresis: (a) sensor setup with axial clamping, (b) cross-sectional and (c) top view of the setup. Hydrogel PVA/PAA, material and geometry parameters as in Table 1.

pipette of a $5 \mathrm{~mL}$ polymer solution, consisting of PVA/PAA, to the prepared surface with respect to the optimum coverage ratio of $x=0.5$ and dried at $295.15 \mathrm{~K}$ for $21600 \mathrm{~s}$. Figure $5 \mathrm{c}$ shows exemplarily the structured hydrogel layer on the bending beam surface. After drying, the polymer was thermally cross-linked in an oven at $393.15 \mathrm{~K}$ for $1200 \mathrm{~s}$ (Arndt et al., 1999).

\section{Deflection measurements of the sensor switch with switching hysteresis}

The hydrogel-coated bending beam was axially clamped in a fixation (Fig. 5). A compression load of $0.04 \mathrm{~N}$ was applied by means of an adjusting screw. For its measurement, a force-measuring device (SAUTER FC 10) was applied on the opposite side of the fixation. The beam-like sensor switch was investigated in a self-made humidity chamber. The humidity was varied using a bubble system and was monitored by means of a humidity sensor (Hygro-Thermometer, $\mathrm{B}+\mathrm{B})$. The deflection of the pre-deflected bending beam was recorded via a CMOS camera (HIKVISION).

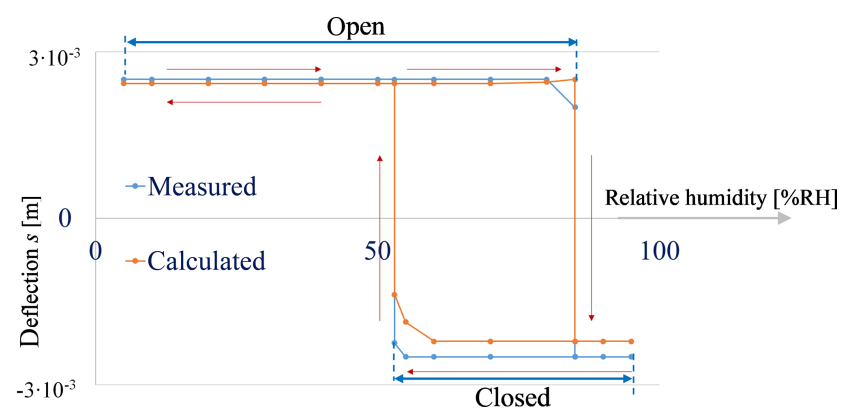

Figure 6. Deflection of the bending beam with structured hydrogel as function of the relative humidity. The resulting switching hysteresis shows two switching points at $53 \% \mathrm{RH}$ and $85 \% \mathrm{RH}$.

\section{Experimental results}

Figure 6 shows the measured deflection of the bending beam as a function of the relative humidity in the range from $5 \% \mathrm{RH}$ up to $95 \% \mathrm{RH}$. Above $85 \% \mathrm{RH}$, the bending beam abruptly switches from the pre-deflected side to the opposite side. At $53 \% \mathrm{RH}$, by changing from the humid to the dry environment, the beam switches back to the initial state demonstrating successfully the switching hysteresis.

To compare the experimental results with the mechanical model, the material properties and the geometry parameters from Table 1 are inserted into Eq. (22). The deflections of the bending beam $s$ as function of the relative humidity are calculated from Eq. (7). In comparison to the measured data, the calculated deflection shows a deviation of $8 \%$, whereas the measured hysteresis width of the switching curve shows a good agreement.

\section{Summary and outlook}

This work deals with the development of a sensor switch with switching hysteresis for humidity sensing. The hysteresis is needed for the safe and reliable switching, i.e. fast opening and closing of the contacts. Based on the beam theory, a mechanical model was derived to calculate the deflection $s$ depending on the degree of coverage $x=l_{B} / l$, i.e. of the 
length ratio of the partly hydrogel-coated bending beam part to the total length of the bending beam. It could be shown that a maximum deflection is achieved when the stiffness of the hydrogel-coated part and the uncoated part are equal, i.e. when the lengths of the coated and uncoated parts of the beam are equal $(x=0.5)$. To include the switching hysteresis in the mechanical model, it was complemented by an axial force $F_{A}$ and the axial compliance $n_{\mathrm{T}}$. From this, it could be shown that the width of the switching hysteresis is adjustable via variation of the geometry parameters, like the thickness or the length of the bending beam. To prove the mechanical model, a beam-like sensor switch was manufactured by means of a metal sheet covered with a hydrogel layer at a coverage degree of 0.5 and the switching hysteresis was successfully demonstrated.

Data availability. The data presented in this article are stored in an internal system according to the guidelines of the DFG.

Author contributions. The authors NG and GG designed and developed the experimental setup. NG and GG developed the mechanical model to describe the sensor switch. NG performed the experiments, analyzed the data, and wrote the initial paper. GG revised the paper and led the project. Both authors approved the final paper.

Competing interests. The authors declare that they have no conflict of interest.

Special issue statement. This article is part of the special issue "Sensors and Measurement Systems 2019". It is a result of the "Sensoren und Messsysteme 2019, 20. ITG-/GMA-Fachtagung", Nuremberg, Germany, 25-26 June 2019.

Acknowledgements. The author would like to thank his supervisor Gerald Gerlach for his support.

Financial support. The authors were supported by the Deutsche Forschungsgemeinschaft (DFG) for financial support within the Hydrogel-based Microsystems Research Training Group (GRK 1865).

Review statement. This paper was edited by Eric Starke and reviewed by two anonymous referees.

\section{References}

AMA: Sector Information: Quarter Reports 2017, available at: http://www.ama-sensorik.de/en/association/sector-information/ quarterreports-2017/, last access: 14 August 2018.
Arndt, K. F., Richter, A., Ludwig, S., Zimmermann, J., Kessler, J., Kuckling, D., and Adler, H. J.: Poly (vinyl alcohol)/poly (acrylic acid) hydrogels, FT-IR spectroscopic characterization of crosslinking reaction and work at transition point, Acta Polymerica, 50, 383-390, 1999.

Bellmann, C.: Leistungslose Feuchteschwellwertschalter (Powerless Humidity Threshold Switch), Dissertation, TU Dresden, 2016.

Bellmann, C., Steinke, A., Frank, T., and Gerlach, G.: Humidity micro switch based on humidity-sensitive polymers, Proc. SPIE 9430, Electroactive Polymer Actuators and Devices (EAPAD), 94302F, 2015.

Chen, L. T., Lee, C. Y., and Cheng, W. H.: MEMS-based humidity sensor with integrated temperature compensation mechanism, Sensors and Actuat., 147, 522-528, 2008.

Dai, M., Picot, O. T., Verjans, J. M., Haan, L. T., Schenning, A. P., Peijs, T., and Bastiaansen, C. W.: Humidity-responsive bilayer actuators based on a liquid-crystalline polymer network, ACS Appl. Mater. Inter., 5, 4945-4950, 2013.

Frank, T., Steinke, A., and Gerlach, G.: Binary ZeroPower Sensors: an alternative solution for power-free energy-autonomous sensor systems, Microsyst. Technol., 18, 1225-1231, 2012.

Gartmann, N., Schütze, C., Ritter, H., and Brühwiler, D.: The effect of water on the functionalization of mesoporous silica with 3aminopropyltriethoxysilane, J. Phys. Chem. Lett., 1, 379-382, 2010.

Huang, W. M., Lei, F., Zhao, M., and Wang, K.: A surface micromachined CMOS MEMS humidity sensor, Micromachines, 6, 1569-1576, 2015.

Jose, J., Shehzad, F., and Al-Harthi, M. A.: Preparation method and physical, mechanical, thermal characterization of poly(vinyl alcohol)/poly(acrylic acid) blends, Polym. Bull., 71, 2787-2802, 2014.

Marschner, U., Gerlach, G., Starke, E., and Lenk, A.: Equivalent circuit models of two-layer flexure beams with excitation by temperature, humidity, pressure, piezoelectric or piezomagnetic interactions, J. Sens. Sens. Syst., 3, 187-211, https://doi.org/10.5194/jsss-3-187-2014, 2014.

Rangelow, I. W., Gerlach, G., Bartuch+, H., Steinke, A., and Roeder, R.: Micromechenical sensors: switch for thresholdrelated switching of electrical contacts, Patentschrift: WO 2009077446 A1 25/06/2009, 2009.

Rittersma, Z. M.: Recent achievements in miniaturised humidity sensors - a review of transduction techniques, Sensors Actuat., 96, 196-210, 2002.

Norm DIN EN 10270-1: Steel wire for mechanical springs - Part 1: Patented cold drawn unalloyed spring steel wire, Beuth Verlag, 2012.

Timoshenko, S. P. and Gere, J. M.: Theory of Elastic Stability, Second edition, Newburyport, Dover Publications, 2012. 